\title{
APPROXIMATIONS OF RUIN PROBABILITY BY DI-ATOMIC OR DI-EXPONENTIAL CLAIMS
}

\author{
By Joshua Babier and Beda Chan \\ University of Toronto, Canada
}

\begin{abstract}
The sensitivity of the ruin probability depending on the claim size distribution has been the topic of several discussion papers in recent ASTIN Bulletins. This discussion was initiated by a question raised by Schmitter at the ASTIN Colloquium 1990 and attempts to make further contributions to this problem. We find the necessary and sufficient conditions for fitting three given moments by diatomic and diexponential distributions. We consider three examples drawn from fire (large spread), individual life (medium spread) and group life (small spread) insurance data, fit them with diatomics and diexponentials whenever the necessary and sufficient conditions are met, and compute the ruin probabilities using well known formulas for discrete and for combination of exponentials claim amounts. We then compare our approximations with the exact values that appeared in the literature. Finally we propose using diatomic and diexponential claim distributions as tools to study the Schmitter problem.
\end{abstract}

\section{KEYWORDS}

Ruin probability; diatomic distribution; diexponential distribution.

\section{INTRODUCTION}

Parametric representation of claim data and exact calculation of ruin probabilities have a long history. In the classical work of CRAMÉR (1955, p. 43), the following claim amount distribution was used to represent data from Swedish non-industry fire insurance covering the years 1948-1951:

$$
p(x)=4.897954 e^{-5.514588 x}+4.503(x+6)^{-2.75}, 0<x<500 .
$$

Exact ruin probabilities were computed by numerically solving

$$
\psi(u)=\frac{\lambda}{c} \int_{0}^{u}[1-P(y)] \psi(u-y) d y+\frac{\lambda}{c} \int_{u}^{\infty}[1-P(y)] d y,
$$

which was a nontrivial numerical task then (CRAMÉR 1955, p. 45). A modern reference for the above integral equation is Exercise 12.11 in BowERs et alii (1986). 
A much easier numerial task even now is to approximate (1) by a distribution for which there is a readily executable formula for its ruin probabilities. In this paper, we choose our approximants from special types of claim amount distributions in which there are recent interests: For combination of exponential distributions, there are the TÄCKLIND (1942) types formulas. See Shiu (1984), Gerber, Goovaerts and KaAs (1987), Dufresne and Gerber (1988) (1989) (1991), and ChAN (1990). For discrete distributions (mixture of atomic distributions), there are the TAKÁCS (1967) type formulas. See Beekman (1968), Shiu (1989) and KaAs (1991). In particular, we consider the special cases of mixture of two atoms (diatomic) and of combination of two exponentials (diexponential).

\section{THREE MOMENT FIT FOR DIATOMIC AND DIEXPONENTIAL DISTRIBUTIONS}

\subsection{Diatomic distributions}

\section{Proposition 1:}

Given mean, variance, and third central moment written as $\mu, \sigma^{2}$, and $\kappa^{3}$, there is a unique diatomic fitting these moments. The locations of the two atoms are

$$
\{\mu-x, \mu+\mathrm{y}\},
$$

and the corresponding probabilities are:

$$
\left\{\frac{\sigma^{2}}{x^{2}+\sigma^{2}}, \frac{x^{2}}{x^{2}+\sigma^{2}}\right\}
$$

where

$$
\begin{aligned}
& x=\frac{\sqrt{\kappa^{6}+4 \sigma^{6}}-\kappa^{3}}{2 \sigma^{2}}, \\
& y=\frac{\sqrt{\kappa^{6}+4 \sigma^{6}}+\kappa^{3}}{2 \sigma^{2}} .
\end{aligned}
$$

Clearly, $x>0$ and $y>0$. In addition, if the given $\mu>0$ and non-negative atoms are desired, then one must have

$$
\kappa^{3} \geq \frac{\sigma^{2}\left(\sigma^{2}-\mu^{2}\right)}{\mu} .
$$

\section{Proof :}

A diatomic distribution has the routine parametrization by probabilities $A, 1-A$ at atoms $x_{1}, x_{2}$. Instead, we choose as the three parameters the mean 
$\mu$ and the locations of the two atoms expressed in (3). The equation of mean $=\mu$ is equivalent to the probabilities at the atoms equal

$$
\left\{\frac{y}{x+y}, \frac{x}{x+y}\right\} \text {. }
$$

The equation of variance $=\sigma^{2}$ is equivalent to

$$
x y=\sigma^{2} \text {. }
$$

With $y=\frac{\sigma^{2}}{x}$, the probabilities (8) can be rewritten as (4). Finally, the equation of third central moment $=\kappa^{3}$ is equivalent to

$$
\frac{\sigma^{2}\left(\sigma^{2}-x^{2}\right)}{x}=\kappa^{3},
$$

which is a quadratic equation in $x$ and has two solutions, one positive and one negative in this case. The positive solution is (5). Use (9) to obtain (6).

To prove (7), observe that for fixed $\mu>0$ and $\sigma^{2}$, as $\kappa^{3}$ goes from the right to the left on the real line, the corresponding $\mu-x$ goes also from the right to the left. The value of $\kappa^{3}$ for which $\mu-x=0$ is given by the right side of (7).

Q.E.D.

Remark: The third central moment equation (10) is best understood as

$$
y-x=\frac{\kappa^{3}}{\sigma^{2}},
$$

since (9) and (11) give a geometric interpretation of $\sigma^{2}$ and $\kappa^{3}$ in terms of the three points: the mean $\mu$ and the two atoms $\mu-x$ and $\mu+y$. Equation (9) says the standard deviation is the geometric mean of $x$ and $y$. Equation (11) says that the asymmetry as measured by $y-x$ is fully responsible for the skewness $\kappa^{3}$, after $\kappa^{3}$ is properly scaled into $\kappa^{3} / \sigma^{2}$. The transformation of $\mu, x, y$ to $k \mu$, $k x, k y$ would change skewness to $k^{3} \kappa^{3}$, but equation (11) would be scale invariant.

\subsection{Diexponential distributions}

Out of two exponentials with parameters $0<\beta \leq \gamma$, we adopt the convention that the smaller of the two parameters is always named $\beta$, and call a distribution with density function

$$
p(x)=A \beta e^{-\beta x}+(1-A) \gamma e^{-\gamma x} \text { for } x \geq 0
$$

a diexponential distribution when and only when $A$ makes the above $p(x)$ a 
(non-negative) density function over $x \geq 0$. The necessary and sufficient conditions for $A$ making $p(x)$ a legitimate density function are:

$$
0 \leq A \leq \frac{\gamma}{\gamma-\beta}
$$

since $A$ negative would lead to $p(x)$ negative for large $x$, and $\frac{\gamma}{\gamma-\beta}<A$ would lead to $p(x)$ negative for small $x$. A member of this three parameter family is traditionally called a mixture of two exponentials when $0 \leq A \leq 1$ and a combination of two exponentials when $1 \leq A \leq \frac{\gamma}{\gamma-\beta}$. Note that when $\beta=\gamma$, $A=0$, or $\mathrm{A}=1$ it degenerates to a single exponential. When $A=\frac{\gamma}{\gamma-\beta}$ we have $p(0)=0$ and it becomes a two parameter family:

$$
p(x)=A \beta e^{-\beta x}-A \beta e^{-\frac{A \beta}{A-1} x} \text { for } x \geq 0, \text { where } 0<\beta, 1<A .
$$

This distribution is the independent sum of two exponentials of parameter $\beta$ and of parameter $\gamma$ and is usually parametrized as:

$$
\begin{aligned}
p(x) & =\frac{\beta \gamma}{\gamma-\beta}\left(e^{-\beta x}-e^{-\gamma x}\right) \text { for } x \geq 0 \\
& =\frac{\gamma}{\gamma-\beta} \beta e^{-\beta x}-\frac{\beta}{\gamma-\beta} \gamma e^{-\gamma x} \text { for } x \geq 0
\end{aligned}
$$

where $0<\beta<\gamma$.

How big is the family of diexponential distributions? Can a diexponential always be found to fit up to the third moment? This question translates into the solution of the following system of equations

$$
\begin{aligned}
& \frac{A}{\beta}+\frac{1-A}{\gamma}=E(X)=\mu, \\
& \frac{A}{\beta^{2}}+\frac{1-A}{\gamma^{2}}=\frac{E\left(X^{2}\right)}{2}=\frac{\sigma^{2}+\mu^{2}}{2}, \\
& \frac{A}{\beta^{3}}+\frac{1-A}{\gamma^{3}}=\frac{E\left(X^{3}\right)}{6}=\frac{\kappa^{3}+3 \sigma^{2} \mu+\mu^{3}}{6} .
\end{aligned}
$$

We shall find that the answer is different from the case of diatomic distributions where any $\mu, \sigma^{2}$, and $\kappa^{3}$ would find a diatomic fit. 
Following the intuition gained from the case of the diatomic distribution, we parametrize the diexponential distribution with three "points", $\mu, b=\frac{1}{\beta}$, and $c=\frac{1}{\gamma}$. Solving (16) for $A$ we get

$$
A=\frac{\beta(\gamma \mu-1)}{\gamma-\beta}=\frac{\mu-c}{b-c},
$$

which allows us to turn (16), (17), and (18) into equations in $\mu, b$, and $c$. Since our points $b$ and $c$ are not atoms, unlike the diatomic case, $1-A$ is allowed to be negative but still gives a genuine probability density function. The convention of $0<\beta \leq \gamma<\infty$ becomes $0<c \leq b<\infty$, and (13) becomes

$$
c \leq \mu \leq b+c \text {. }
$$

Consider

$$
\begin{aligned}
\sigma^{2} & =2 \mu b+2 \mu c-2 b c-\mu^{2} \\
& =\mu^{2}+(b-c)^{2}-(\mu-c)^{2}-(b-\mu)^{2} \\
& =\mu^{2}-2\{b c-(b+c-\mu) \mu\}
\end{aligned}
$$

where the first equality is obtained by solving (17) for $\sigma^{2}$ and the other two are algebraic reformulations. For $\mu$ fixed and $0<c \leq \mu \leq b$ (mixture of exponentials, i.e., $0 \leq A \leq 1),(21)$ tells us that $\sigma^{2}$ is at least $\mu^{2}$ and gets close to $\mu^{2}$ when $c \rightarrow \mu$ and $\mu \leftarrow b$. For $\mu$ fixed and $0<b<\mu \leq b+c$ (combination of exponentials, i.e., $1<A \leq \frac{b}{b-c}$ ), (22) tells us that $\sigma^{2}$ is at most $\mu^{2}$ and at least $\frac{\mu^{2}}{2}$ because $\{\cdot\}$ is always non-negative and is at most $\frac{\mu^{2}}{4}$. The last statement is verified by the observation that $b+c=(b)+(c)=(b+c-\mu)+(\mu)$ and the maximum of $\{\cdot\}$ as a function of the two variables $b$ and $c$ is reached at $\mu \leftarrow b+c$ and $b \leftrightarrow c$.

For fixed $\mu$, we found that it is necessary for $\sigma^{2}$ to be big $\left(>\mu^{2}\right)$ to fit a mixture; and one would guess $\kappa^{3}$ must not to be too small. The exact lower bound of $\kappa^{3}$ will be determined later. It is necessary for $\frac{\mu^{2}}{2}<\sigma^{2}<\mu^{2}$ to fit a combination; and one would guess $\kappa^{3}$ must be in an interval too. The exact interval for $\kappa^{3}$ will be determined later. For $\sigma^{2}=\mu^{2}$, (21) and (22) both tell us that it must be a single exponential, thus (ii) below is proved. For $\sigma^{2}=\frac{\mu^{2}}{2}$, 
(22) tells us that we must have $c=b=\frac{\mu}{2}$, thus (iv) below is proved. We have just proved most of part $\mathrm{A}$ in the following proposition.

\section{Proposition 2:}

A. Necessary and sufficient conditions for finding a fit: For a fixed $\mu>0$,

(i) for $\sigma^{2}>\mu^{2}$, if $\kappa^{3}>\frac{\mu^{4}+3 \sigma^{4}}{2 \mu}$, then a mixture of exponentials with $A<1$ fits, if $\kappa^{3} \leq \frac{\mu^{4}+3 \sigma^{4}}{2 \mu}$, then there is no diexponential that fits;

(ii) for $\sigma^{2}=\mu^{2}$, if $\kappa^{3}=\frac{\mu^{4}+3 \sigma^{4}}{2 \mu}=2 \mu^{3}$, then the fitting diexponential degenerates into a single exponential $(1 / \mu)$, any other value of $\kappa^{3}$ has no diexponential fit;

(iii) for $\frac{\mu^{2}}{2}<\sigma^{2}<\mu^{2}$,

if $6 \mu \sigma^{2}-4 \mu^{3}+\sqrt{18\left(\mu^{2}-\sigma^{2}\right)^{3}}<\kappa^{3}<\frac{\mu^{4}+3 \sigma^{4}}{2 \mu}$,

then a combination of exponentials with $A>1$ fits, if $\kappa^{3} \leq 6 \mu \sigma^{2}-4 \mu^{3}+\sqrt{18\left(\mu^{2}-\sigma^{2}\right)^{3}}$ or $\frac{\mu^{4}+3 \sigma^{4}}{2 \mu} \leq \kappa^{3}$, then there is no diexponential that fits;

(iv) for $\sigma^{2}=\frac{\mu^{2}}{2}$, if $\kappa^{3}=6 \mu \sigma^{2}-4 \mu^{3}+\sqrt{18\left(\mu^{2}-\sigma^{2}\right)^{3}}=\frac{\mu^{3}}{2}$,

then the fitting diexponential degenerates into a single gamma $(2,2 / \mu)$, any other value of $\kappa^{3}$ has no diexponential fit;

(v) for $\sigma^{2}<\frac{\mu^{2}}{2}$, there is no diexponential that fits the given moments. 
B. The fit: When the three moments $\mu, \sigma^{2}, \kappa^{3}$ satisfy the above conditions to give a fitting diexponential, the appropriate parameters are:

(23) $\frac{1}{\beta}, \frac{1}{\gamma}=\frac{\kappa^{3}-2 \mu^{3} \pm \sqrt{\kappa^{6}-4 \kappa^{3} \mu\left(3 \sigma^{2}-2 \mu^{2}\right)+18 \sigma^{6}-18 \sigma^{4} \mu^{2}+6 \sigma^{2} \mu^{4}-2 \mu^{6}}}{6\left(\sigma^{2}-\mu^{2}\right)}$

$$
A=\frac{\beta(\gamma \mu-1)}{\gamma-\beta} .
$$

\section{Proof :}

The bounds for $\kappa^{3}$ will be found naturally after we solve (16), (17), and (18) and establish B. Substitute (19) into (17) and (18) and write everything in terms of $\mu, b$, and $c$. Solve the second moment equation for $b$ and substitute this back in to the third moment equation for $c$. This is a quadratic equation which we solve to get solutions $c_{1}, c_{2}$. Substitute back to get $b_{1}, b_{2}$. With the algebraic symmetry of $b$ and $c$, it is not surprising that we found $\left\{c_{1}, b_{1}\right\}$ and $\left\{c_{2}, b_{2}\right\}$ are the same set of two numbers. By the convention of $\beta \leq \gamma$, we name the smaller number $c$ and the bigger number $b$. The formulas in $\mathrm{B}$ are thus established.

The mysterious bounds in A are determined by studying (23). The fact that (23) must give positive values leads to the question of when is the right side of (23) zero:

$$
\left(\kappa^{3}-2 \mu^{3}\right)^{2}=\kappa^{6}-4 \kappa^{3} \mu\left(3 \sigma^{2}-2 \mu^{2}\right)+18 \sigma^{6}-18 \sigma^{4} \mu^{2}+6 \sigma^{2} \mu^{4}-2 \mu^{6},
$$

which is a linear equation in $\kappa^{3}$. Solving for $\kappa^{3}$ gives

$$
\kappa^{3}=\frac{\mu^{4}+3 \sigma^{4}}{2 \mu} \text {. }
$$

The fact that (23) must not give complex numbers leads to the equation of the expression under the square root sign in (23) equals zero:

$$
\kappa^{6}-4 \kappa^{3} \mu\left(3 \sigma^{2}-2 \mu^{2}\right)+18 \sigma^{6}-18 \sigma^{4} \mu^{2}+6 \sigma^{2} \mu^{4}-2 \mu^{6}=0,
$$

which is quadratic in $\kappa^{3}$. The two roots for $\kappa^{3}$ are

$$
\kappa^{3}=6 \mu \sigma^{2}-4 \mu^{3} \pm \sqrt{18\left(\mu^{2}-\sigma^{2}\right)^{3}} .
$$

Of the two roots, the one taking the + sign is bigger and determines the boundary that $\kappa^{3}$ must not go below.

Q.E.D.

\section{RUIN PROBABILITIES FOR DIATOMIC AND DIEXPONENTIAL DISTRIBUTIONS}

The ruin probability formula for a discrete claim amount distribution has been given by SchmitTER (1990). See KAAS (1991, p. 136). For similar formulas see 
SHIU (1989). Proof for the atomic case and a reference to FELLER (1971) are found in SHIU (1987). We list the ruin probability formula for diatomic claim amounts below for use in Section 4:

$$
\begin{aligned}
& \psi(u)=1-\frac{\theta}{1+\theta} \sum_{k_{1}, k_{2}}(-z)^{k_{1}+k_{2}} e^{z} \frac{p_{1}^{k_{1}} p_{2}^{k_{2}}}{k_{1} ! k_{2} !}, \\
& \text { where } z=\frac{\left(u-k_{1} x_{1}-k_{2} x_{2}\right)_{+}}{(1+\theta) \mu} .
\end{aligned}
$$

The theory of ruin probability for mixture and combination of exponentials is well known. See SHIU (1984), Dufresne and Gerber (1988), (1989), (1991), and ChAN (1990). In the case when there are only two exponentials, the adjustment coefficient equation

$$
(1+\theta) \mu=\frac{M_{X}(r)-1}{r}
$$

is quadratic and has solutions:

$$
R, r_{2}=\frac{1}{2}\left(\beta+\gamma-\frac{1}{\mu(1+\theta)} \mp \sqrt{\left.\left(\frac{1}{\mu(1+\theta)}-(\beta+\gamma)\right)^{2}-\frac{4 \beta \gamma \theta}{1+\theta}\right)}\right.
$$

and

$$
\psi(u)=C_{1} e^{-R u}+C_{2} e^{-r_{2} u}
$$

where $C_{1}, C_{2}$ are found by the Täcklind formula $\left(r_{1}=R\right)$ :

$$
C_{k}=\prod_{\substack{i \neq k \\ i=1}}^{2} \frac{r_{i}}{r_{i}-r_{k}} \prod_{i=1}^{2} \frac{\beta_{i}-r_{k}}{\beta_{i}} k=1,2 .
$$

\section{DIATOMIC AND DIEXPONENTIAL AS APPROXIMANTS}

In this section, we study three claim amount distributions and compute ruin probabilities of approximating diatomics and approximating diexponentials with matching first three moments and compare the approximations with the exact values of $\psi(u)$. In the first example (Cramér's fire) the claim amount distribution has a large spread, none of the approximations is very close to the exact value. Along with the first example we discuss the run-off error problem encountered in the Takács type formulas. In the second example (Reckin, Schwark, and Snyder's individual life) the claim amount distribution has a medium spread, both the diatomic and the diexponential give good approximations. In the third example (Mereu's group life) the claim amount distribution has a small spread, the diatomic gives an excellent approximation, but the spread is so small that there is no diexponential fit. 
Example 1: We consider Cramér's fire insurance data, the one mentioned in the introduction. In the following table, the exact values of $\psi(u)$ for $\theta=0.3$, and the values by the Cramér-Lundberg approximation are taken from CrAmÉr (1955, p. 45). The values by the Beekman-Bowers approximation are taken from BEEKMAN $(1969$, p. 279). The ruin probability formula for diatomic claims, (24), leads to convergence problems when $u$ is large. Our exprience echoes with that reported in SEAH $(1990, \S 4)$. For values of $u$ close to and above 30 times $\mu$, run-off errors take over and we obtain probabilities less than zero or greater than one. These problematic values are indicated by ${ }^{* *}$ below. In Table 1 , the approximating diatomic has atoms $\{.7657175616,181.1382584\}$ and probabilities $\{.9987011192, .001298880855\}$ as found by (3), (4), (5), and (6). The approximating diexponential has $\frac{1}{\beta}=60.75201696, \frac{1}{\gamma}=.6552147239$, and $A=.005737165094$ as found by (23) and (19).

TABLE 1

CRAMÉr's Fire InSURANCE

$\mu=1, \sigma^{2} / \mu^{2}=42.20323069, \kappa^{3} / \sigma^{3}=27.69286626$

\begin{tabular}{lccccccccc}
\hline$u$ & $\psi(u)$ & $\mathrm{CL}$ & $\mathrm{BB}$ & diatom. & diep. & $\mathrm{CL} / \psi(u)$ & $\mathrm{BB} / \psi(u)$ & dia. $/ \psi(u)$ & die. $/ \psi(u)$ \\
\hline 20 & .5039 & .4524 & .5140 & .4133 & .4666 & 0.898 & 1.020 & 0.820 & 0.926 \\
40 & .3985 & .3904 & .4079 & $* *$ & .4010 & 0.980 & 1.028 & $* *$ & 1.006 \\
60 & .3280 & .3370 & .3369 & $* *$ & .3447 & 1.027 & 1.027 & $* *$ & 1.051 \\
80 & .2757 & .2909 & .2812 & $* *$ & .2962 & 1.055 & 1.020 & $* *$ & 1.074 \\
100 & .2346 & .2511 & .2369 & $* *$ & .2546 & 1.070 & 1.010 & $* *$ & 1.085 \\
\hline
\end{tabular}

Example 2: In this example, we consider the individual life insurance data from Reckin, Schwark, and Snyder (1984). This claim amount distribution was studied as Example 3 in SEACH (1990), from where we took the exact values of $\psi(u)$. The claim amount $X$ is discrete with support $\{1,2,3,4,5,7,8,10,12,13,15,16\}$ and probabilities (in order) $\{.5141, .3099, .0639$, $.0220, .0194, .0096, .0276, .0036, .0041, .0019, .0013, .0226\}$. Since the claim amount distribution is dispersed enough, we have a diexponential fit by (i) of Proposition 2.

TABLE 2.1

$\psi(u)$ By SEAH FOR RSS's INDIVIDUAL LiFE INSURANCE DATA $\mu=2.2896, \sigma^{2} / \mu^{2}=1.43257300, \kappa^{3} / \sigma^{3}=3.60560786$

\begin{tabular}{llllll}
\hline \hline & $\theta=.1$ & $\theta=.2$ & $\theta=.3$ & $\theta=.4$ & $\theta=.5$ \\
\hline$u=0$ & .909091 & .833333 & .769231 & .714286 & .666667 \\
$u=10$ & .644361 & .450722 & .334890 & .260412 & .209732 \\
$u=20$ & .469129 & .254324 & .152965 & .099371 & .068466 \\
$u=30$ & .341528 & .143813 & .070341 & .038430 & .022840 \\
$u=40$ & .248408 & .081101 & .032173 & .014735 & .007526 \\
$u=50$ & .180700 & .045752 & .014725 & .005654 & .002482 \\
\hline
\end{tabular}


TABLE 2.2

(DIATOMIC APPROXIMANT) $/ \psi(u)$ FOR RSS'S DATA

The approximating diatomic has atoms $\{1.580450117,12.887964915\}$ and probabilities $\{.9372389245$, $.06276107517\}$ as found by (3), (4), (5), and (6).

\begin{tabular}{llllll}
\hline$=$ & $\theta=.1$ & $\theta=.2$ & $\theta=.3$ & $\theta=.4$ & $\theta=.5$ \\
\hline$u=0$ & 1 & 1 & 1 & 1 & 1 \\
$u=10$ & 1.013 & 1.029 & 1.045 & 1.060 & 1.073 \\
$u=20$ & 1.003 & 1.007 & 1.012 & 1.015 & 1.018 \\
$u=30$ & 1.001 & 1.000 & 0.996 & 0.990 & 0.981 \\
$u=40$ & 1.001 & 0.999 & 0.992 & 0.982 & 0.968 \\
$u=50$ & 1.001 & 0.997 & 0.988 & 0.974 & 0.957 \\
\hline
\end{tabular}

TABLE 2.3

(DIEXPONENTIAL APPROXIMANT) $/ \psi(u)$ FOR RSS'S DATA

The approximating diexponential has $\frac{1}{\beta}=5.448377581, \frac{1}{\gamma}=1.930653556$, and $A=.1020393986$ as found by (23) and (19).

\begin{tabular}{llllll}
\hline \hline & $\theta=.1$ & $\theta=.2$ & $\theta=.3$ & $\theta=.4$ & $\theta=.5$ \\
\hline$u=0$ & 1 & 1 & 1 & 1 & 1 \\
$u=10$ & 0.997 & 0.984 & 0.966 & 0.947 & 0.928 \\
$u=20$ & 0.994 & 0.985 & 0.979 & 0.978 & 0.984 \\
$u=30$ & 0.995 & 0.991 & 0.997 & 1.016 & 1.047 \\
$u=40$ & 0.996 & 1.000 & 1.022 & 1.066 & 1.132 \\
$u=50$ & 0.998 & 1.009 & 1.048 & 1.119 & 1.224 \\
\hline
\end{tabular}

Example 3: In this example, we consider the group insurance data from Mereu (1972). This claim amount distribution was studied as Example 2 in SEAH (1990), from where we took the exact values of $\psi(u)$. The claim amount $X$ is discrete with support $\{4,6,8,10,12,14,16,20,25\}$ and probabilities (in order) $\{.15304533960, .07882237436, .11199119040, .10432698260, .09432769021$, $.10925807990, .09727308107, .18073466720, .07022059474\}$.

TABLE 3.1

$\psi(u)$ by Seah for Mereu's Group Life Insurance Data $\mu=12.61243786, \sigma^{2} / \mu^{2}=0.25079144, \kappa^{3} / \sigma^{3}=0.30556145$

\begin{tabular}{lllll}
\hline$=$ & $\theta=.25$ & $\theta=.5$ & $\theta=.75$ & $\theta=.1$ \\
\hline$u=0$ & .8 & .666667 & .571429 & .5 \\
$u=25$ & .433995 & .232316 & .141606 & .094198 \\
$u=50$ & .222739 & .072766 & .030113 & .014607 \\
$u=75$ & .114114 & .022685 & .006349 & .002236 \\
$u=100$ & .058463 & .007072 & .001339 & .000342 \\
\hline
\end{tabular}


TABLE 3.2

(Diatomic approximant) $/ \psi(u$ for Mereu's Group Life Insurance Data The approximating diatomic has atoms $\{7.187946466,19.96691435\}$ and probabilities $\{.5755141225$, $.4244858774\}$ as found by (3), (4), (5), and (6).

\begin{tabular}{lllll}
\hline \hline & $\theta=.25$ & $\theta=.5$ & $\theta=.75$ & $\theta=.1$ \\
\hline$u=0$ & 1 & 1 & 1 & 1 \\
$u=25$ & 0.9995 & 0.9992 & 0.9986 & 0.9977 \\
$u=50$ & 1.0003 & 1.0004 & 0.9988 & 0.9962 \\
$u=75$ & 1.0000 & 0.9978 & 0.9929 & 0.9857 \\
$u=100$ & 0.9997 & 0.9962 & 0.9888 & 0.9795 \\
\hline
\end{tabular}

The diatomic approximant is producing excellent values! Since the variance is quite small, there is no diexponential fit as indicated by Proposition 2, (v). Note that because the approximating claims distribution has the same mean and variance as the orignial, the non-ruin probabilities are overestimated as well.

\section{THE SCHMITTER PROBLEM}

The Schmitter problem asks: Given $\theta, u, \mu, \sigma^{2}$, and the range $[0, b]$, is there a distribution with support on $[0, b]$ which would maximize the ruin probability $\psi(u)$ ? See Brockett, Goovaerts, and TAYlor (1991) and KaAS (1991). Schmitter's conjecture of diatomic being the one giving the extremal ruin probabilitity inspires us to use diatomics as approximants. The conjecture, however, has been disproved by KAAS (1991).

The general question is the stability of $\psi(u)$ when $p(x)$ is under perturbation. Schmitter specialized to the question of extreme value of $\psi(u)$ for fixed $\theta$, $u, \mu, \sigma^{2}$, and range $[0, b]$. We would ask another specialized question: Find the extreme value of $\psi(u)$ for fixed $\theta, u, \mu, \sigma^{2}$, and $\kappa^{3}$. Like the Schmitter problem, our question may not have a complete solution. Our question is related to the practical problem: When the true claim amount distribution is represented by the sample, which is a discrete distribution, or is parametrized, for example, as a mixture of exponentials, how robust is the ruin probability? In this paper we have found computational tools to address the stability of $\psi(u)$ when $p(x)$ is diatomic or diexponential with first three given moments.

\section{ACKNOWLEDGEMENT}

The authors have benefitted from conversations and discussions with Professors NanCY REID of Toronto and ERIC SEAH of Manitoba. The first author thanks the Natural Sciences and Engineering Research Council of Canada for a University Undergraduate Research Award. We thank the Actuarial Computing Laboratory and CQUEST (Computers in Quantitative and Empirical Science Teaching) at the University of Toronto for computing facilities. We also thank the referees and a co-editor especially for suggestions clarifying the proofs of Propositions 1 and 2. 


\section{REFERENCES}

BeEkman, J.A. (1968) Collective risk results. TSA 20, 182-199.

BeEkMAN, J.A. (1969) A ruin function approximation. TSA 21, 41-48, 275-282.

Bowers, N. L. Jr., Gerber, H. U., Hickman, J. C., Jones, D. A. and Nesbitt, C. J. (1986) Actuarial Mathematics. Society of Actuaries, Schaumburg, Illinois.

Brockett, P., Goovaerts, M. and Taylor, G. (1991) The Schmitter problem. ASTIN Bulletin 21, $129-132$.

Chan, B. (1990) Ruin probability for translated combination of exponential claims. ASTIN Bulletin 20, $113-114$.

Cramer, H. (1955) Collective Risk Theory. Skandia Jubilee Volume, Nordiska Bokhandeln, Stockholm.

Dufresne, F. and Gerber, H. U. (1988) The probability and severity of ruin for combination of exponential claim amount distribution and their translations. Insurance: Mathematics and Economics 7, 75-80.

Dufresne, F. and Gerber, H. U. (1989) Three methods to calculate the probability of ruin. ASTIN Bulletin 19, 71-90.

Dufresne, F. and Gerber, H.U. (1991) Rational ruin problems - a note for the teacher. Insurance: Mathematics and Economics 10, 21-29.

FELLER, W. (1971) An Introduction to Probability Theory and Its Applications. Vol. Il, 2nd ed. Wiley, New York.

Gerber, H. U., Goovaerts, M.J. and KaAs, R. (1987) On the probability and severity of ruin. ASTIN Bulletin 17, 151-163.

KAAS, R. (1991) The Schmitter problem and a related problem : a partial solution. ASTIN Bulletin 21, $133-146$.

Mereu, J.A. (1972) An algorithm for computing expected stop-loss claims under a group life contract. TSA 24, 311-320.

Reckin, G.E., Schwark, D.J. and Snyder, J.B. II (1984) Practical applications of the ruin function. TSA 36, $453-477$.

SCHMITTER, H. (1990) The ruin probability of a discrete claims distribution with a finite number of steps. XXII ASTIN Colloquium, Montreux, Switzerland.

SEAH, E. S. (1990) Computing the probability of eventual ruin. TSA 42, 421-446.

SHIU, E.S.W. (1984) Discussion on "Practical applications of the ruin function". TSA 36 , $480-486$.

SHIU, E.S.W. (1987) Convolution of uniform distribution and ruin probability. Scandinavian Actuarial Journal 70, 191-197.

SHIU, E.S.W. (1989) Ruin probability by operational calculus. Insurance: Mathematics and Economics 8, 243-250.

Täcklind, S. (1942) Sur le risque de ruine dans des jeux inéquitables. Scandinavian Actuarial Journal, 1-42.

TAKÁCS, L. (1967) Combinatorial Methods in the Theory of Stochastic Processes. Wiley, New York.

\section{Joshua Babier and Beda Chan \\ Department of Statistics, 100 St George Street, University of Toronto, Toronto, Ontario, Canada M5S 1 A1.}

